\title{
Competências Socioemocionais: Análise da Produção Científica Nacional e Internacional
}

\section{Socioemotional Skills: Analysis of Brazilian and International Scientific Production}

\author{
Maristela Volpe dos Santos' ${ }^{1}$ Talita Fernanda da Silva², Gabriela Fabbro Spadari3, \\ Tatiana de Cássia Nakano 4
}

\begin{abstract}
Resumo
As competências socioemocionais têm sido, cada vez mais, foco de estudos desenvolvidos nas mais diversas áreas. Considerando-se sua relevância nos resultados pessoais, educacionais e laborais, o presente estudo buscou realizar uma análise da produção científica nacional e internacional na temática. Uma consulta aos bancos de dados nacionais (SciELO, Pepsic e Banco de Teses e Dissertações da Capes) e ao banco internacional (Wiley Online Library), no período de 2011 a 2015 , apontou a existência de 67 artigos, sendo a maior parte deles provenientes da base internacional $(\mathrm{n}=50)$. Os resultados mostraram, de maneira geral, oscilação no interesse pela temática ao longo do período analisado, focados na área da Psicologia do Desenvolvimento e Educação. Cinco temas predominantes foram encontrados: aspectos sociais, características de personalidade, fatores de risco e proteção no desenvolvimento, indicadores de sucesso escolar e profissional e aspectos cognitivos. A maior parte dos trabalhos eram de natureza empírica e fizeram uso de 23 diferentes instrumentos, notadamente em amostras de crianças. O número pequeno de estudos nacionais apontou para uma temática que merece ser mais bem explorada, notadamente pela Psicologia e pela área da avaliação psicológica.

Palavras-chave: Competências socioemocionais. Avaliação psicológica. Competências não cognitivas. Personalidade.
\end{abstract}

\begin{abstract}
Socioemotional competences have increasingly been the focus of studies carried out in several areas. Considering their relevance in personal, educational and professional results, the present study sought to make an analysis of the Brazilian and international scientific production in this area. A search in national databases (SciELO, Pepsic and Banco de Teses e Dissertações da Capes) and an international database (Wiley Online Library), from 2011 to 2015, pointed out the existence of 67 articles, most of which from the international database $(n=50)$. Overall, the results showed an oscillation in the interest in the theme over the analyzed period, focused on the area of Developmental and Educational Psychology. Five predominant themes were found: social aspects, personality characteristics, risk and protection developmental factors, indicators of school and professional success, and cognitive aspects. Most of the studies were empirical and made use of 23 different instruments,
\end{abstract}

\footnotetext{
${ }^{1}$ Pontifícia Universidade Católica de Campinas, Campinas, Brasil. E-mail: maristela.volpe@gmail.com

${ }^{2}$ Pontifícia Universidade Católica de Campinas, Campinas, Brasil. E-mail: talita_fs@ig.com.br

${ }^{3}$ Pontifícia Universidade Católica de Campinas, Campinas, Brasil. E-mail: gfspadari@gmail.com

${ }^{4}$ Programa de Pós-Graduação em Psicologia da Pontifícia Universidade Católica de Campinas, Campinas, Brasil. E-mail: tatiananakano@hotmail.com
} 
notably in samples of children. The small number of Brazilian studies suggests a topic that deserves to be better explored, notably by Psychology and the field of psychological assessment.

Keywords: Socioemotional skills. Psychological assessment. Non-cognitive skills. Personality. 


\section{Introdução}

Atualmente, as exigências do mundo moderno acarretaram significativas mudanças no que se refere ao preparo de crianças e jovens para os desafios do século XXI. Tais mudanças envolvem, principalmente, o oferecimento de condições que visam ao desenvolvimento das competências necessárias para o sucesso acadêmico, profissional e pessoal. Tais competências vêm sendo classificadas em duas vertentes: as reconhecidas e mensuradas pelos sistemas educativos, chamadas de competências cognitivas (relacionadas às disciplinas curriculares e aspectos cognitivos), bem como aquelas que não fazem parte do currículo, porém tão importantes quanto, chamadas de competências socioemocionais (Santos \& Primi, 2014).

Também conhecidas como competências para o século XXI, tais habilidades têm sido, cada vez mais, destacadas na literatura científica internacional (Durlak, Weissberg, Dymnicki, Taylor \& Schellinger, 2011). Isso porque o resultado de uma série de estudos, conduzidos pelas mais diferentes áreas de conhecimento, têm demonstrado que indivíduos com altos níveis de desenvolvimento socioemocional apresentam, dentre outros resultados, maiores indicadores de bem-estar, menor prevalência de sintomas depressivos e maiores índices de satisfação nos relacionamentos interpessoais (Eisenberg, 2006; Guerra \& Bradshaw, 2008; Goldenberg, Matheson \& Mantler, 2006; Lopes, Salovey, Coté, Beers \& Petty, 2005), assim como em indicadores do funcionamento da vida em geral (Lipnevich \& Roberts, 2012).

Diferentes contextos têm ressaltado a importância dessas competências socioemocionais. Notadamente, no contexto organizacional, as pesquisas têm demonstrado que, quando desenvolvidas, tais competências podem atuar no sentido de prevenir o absentismo, infrações disciplinares, desemprego e baixo salário (Heckman \& Rubinstein, 2001; Heckman, Stixrud \& Urzua, 2006; Lindqvist \& Vestman, 2011; MacCann, Duckworth \& Roberts, 2009). Além disso, auxiliariam na estabilidade laboral e produtividade, exercendo até mesmo influência sobre as habilidades cognitivas geradoras de sucesso (Bowles \& Gintis, 1976; Klein, Spady \& Weiss, 1991; Thiel \& Thomsen, 2013). Também no ambiente educacional, destaque tem sido dado à contribuição das competências socioemocionais para o aprimoramento do processo de ensino-aprendizagem, promoção do sucesso escolar, prevenção de problemas de aprendizagem, insucesso acadêmico (Abed, 2014), frequência e até mesmo horas adicionais de estudo (Delaney, Harmon \& Ryan, 2013).

Dados os motivos apontados, o que se tem visto, notadamente nas últimas décadas, é que essa temática tem sido valorizada em diferentes áreas do conhecimento, com destaque para a economia, sociologia, psicologia e educação (Heckman, Stixrud \& Urzua, 2006). Se, historicamente, as competências cognitivas foram valorizadas, de forma quase exclusiva como elemento essencial para o sucesso pessoal e profissional, a valorização da combinação dessas habilidades com as competências socioemocionais têm ganhado espaço nas políticas públicas e educacionais de diversos países, dada sua relevância nos mais diferentes contextos.

Os dois tipos de competências interagem entre si e possibilitam sucesso em diferentes âmbitos, sendo, ambas, vias importantes por meio das quais a educação tem afetado, de forma positiva, uma série de resultados sociais (Miyamoto, Huerta, Kubacka, Ikesako \& Oliveira, 2015). As competências já reconhecidas e mensuradas pelos sistemas educativos, relacionadas principalmente ao letramento, assim 
como ao acúmulo de conhecimento cristalizado, constituem os chamados aspectos cognitivos, usualmente avaliados por meio de testes de desempenho (Santos \& Primi, 2014). Outros fatores que usualmente não fazem parte do currículo intencional das escolas, mas que são igualmente importantes para o desenvolvimento do ser humano, os quais envolvem traços, comportamentos e habilidades que não são medidos nos testes cognitivos convencionais, tais como perseverança, conscienciosidade, autocontrole, confiança, atenção, autoestima e autoeficácia, capacidade de resistência às adversidades, abertura à experiência, empatia, humildade, tolerância a opiniões diversas e a capacidade de envolver-se produtivamente na sociedade, as quais têm se mostrado valorizadas no mercado de trabalho, na escola e na sociedade em geral (Krauz, Heckman, Diris, Weel \& Borghans, 2015), podem ser consideradas variáveis não cognitivas (Lee \& Shute, 2009).

Considerando-se que pesquisas conduzidas por economistas, psicólogos e educadores nas últimas décadas têm revelado que as competências não cognitivas assumem papel tão importante quanto as competências cognitivas para a obtenção de bons resultados em diferentes áreas do bem-estar individual e social (Santos \& Primi, 2014), o conhecimento da área mostra-se fundamental dada à possibilidade de estudo da relação entre desenvolvimento socioemocional, aprendizagem e formação de pessoas em sua integralidade. Dadas as evidências de que tais competências atuam como preditoras de importantes índices relacionados ao desempenho educacional e promoção do bem-estar na idade adulta, a importância de sua investigação se justifica, notadamente se considerarmos que o domínio de aspectos motivacionais e afetivos em si e nos outros, assegura a aprendizagem, o desenvolvimento e a transferência dessas capacidades para outros contextos (Gondim, Morais \& Brantes, 2013).

As competências socioemocionais são definidas como um conjunto de traços, comportamentos e habilidades que incluem tipicamente: 1. Variáveis como atitude, valores, interesse e curiosidade, 2. Variáveis de temperamento e personalidade como abertura a novas experiências, amabilidade, conscienciosidade, extroversão e estabilidade emocional, 3. Variáveis sociais como liderança, sensibilidade social e habilidade de trabalhar com outros, 4. Construtos voltados à autoeficácia, autoestima e identidade pessoal, 5 . hábitos de trabalho, tais como esforço, disciplina, persistência e manejo de tempo, assim como 6 . Emoções direcionadas a tarefas específicas, notadamente entusiasmo e ansiedade (Abed, 2014; Lee \& Shute, 2009).

Ademais, Santos e Primi (2014) também destacam a persistência, resiliência, comprometimento, capacidades relacionadas ao aprendizado, formas de pensar, comunicação, colaboração, autonomia, dentre outras que vêm sendo valorizadas internacionalmente, notadamente pela Organisation for Economic Cooperation and Development e European Reference Framework. Diante da amplitude de elementos que podem ser considerados como constituintes das competências socioemocionais, o que se pode ver é que tal construto vem sendo compreendido de forma bastante ampla e multidimensional, envolvendo aspectos de natureza social e emocional.

De modo geral, uma tendência que se faz notar, quando se abordam as diferentes características relacionadas às competências socioemocionais, na literatura científica nacional e internacional, é o agrupamento dessas características 
e das evidências disponíveis dentro do modelo dos cinco grandes fatores da personalidade, também chamado de Big Five (Santos \& Primi, 2014). Pesquisas internacionais recentes têm se embasado na ideia de que as competências socioemocionais apresentam uma relação estreita com traços de personalidade, os quais parecem exercer significativa influência nas competências socioemocionais (Lipnevich \& Roberts, 2012; Mervielde \& De Fruyt, 2002; Milici, Alcalay, Berger \& Álamos, 2013), em conjunto com outros aspectos, notadamente os sociais, emocionais e hábitos.

Indiferente ao seu reconhecimento, cada vez maior, visualizado desde a década de 1970 (Messick, 1979), uma série de dificuldades ainda se fazem presentes na temática, as quais envolvem: 1. Ausência de consenso em relação à terminologia, também chamadas de características psicossociais, disposição, fatores de aptidão acadêmica/escolar, competências socioafetivas, pessoais e competências do século XXI ((Lipnevich \& Roberts, 2012). 2. Uma carência de informação sobre quais competências devem ser promovidas, bem como sobre a melhor forma de fazê-lo (Miyamoto et al., 2015), aliada à ausência de avaliações rigorosas ou em longo prazo acerca das intervenções realizadas para o desenvolvimento de tais competências. 3. Questionamentos acerca de quais seriam as competências socioemocionais que devem ser alvo dos processos educacionais nas escolas. Como mensurar o impacto dessas ações? Quais são os desafios envolvidos na promoção das habilidades socioemocionais no espaço escolar? (Abed, 2014). 4. A limitação no número de iniciativas, práticas, políticas públicas, currículos ou abordagens centrais voltadas à promoção do desenvolvimento socioemocional (Papadopoulou et al., 2014). 5. Limitação no número de instrumentos de medidas voltados à mensuração das competências socioemocionais (Santos \& Primi, 2014).
Dentre as dificuldades relatadas, a pouca consciência acerca de como elas funcionam, meios de estimulação e os esforços para medi-las e fomentá-las (Miyamoto et al., 2015), aliada ao pouco esforço que tem sido dedicado ao seu desenvolvimento intencional e à avaliação da efetividade das intervenções com objetivo de promovê-las (Santos \& Primi, 2015), tem contribuído para que a temática ainda se mostre muito recente em nosso país e pouco explorada, seja na prática escolar ou nas pesquisas acadêmicas.

Diante do exposto, o presente estudo teve como objetivo central a realização de uma análise da produção científica nacional e internacional a respeito do construto competência socioemocional publicada nos últimos cinco anos. Mais especificamente, buscou 1. Identificar tendências nas pesquisas por meio da verificação de seu ano de publicação, área de conhecimento, tipo de estudo, tema enfocado, instrumento utilizado e população estudada, bem como 2. Identificar tendências e lacunas ainda presentes na temática.

\section{Método}

\section{Material}

Para o estudo foram analisados 67 resumos de estudos científicos publicados no período de 2011 a 2015 nas bases de dados nacionais SciELO, Pepsic, Banco de Teses e Dissertações da Capes e uma base de dados internacional, Wiley Online Library, cuja temática voltava-se à investigação das competências socioemocionais na área da Psicologia.

Foram selecionados e analisados 17 resumos nacionais, sendo seis provenientes da SciELO, dez dos periódicos da Capes e um do Banco de Teses e Dissertações da Capes, além de 50 resumos oriundos da base de dados internacional.

\section{Procedimentos}


A partir da combinação de três palavraschaves "socioemotional", "skills" e "psychology", os resultados foram limitados àqueles publicados nos últimos cinco anos (2011 a 2015). Um total de 17 trabalhos científicos foram encontrados nos bancos nacionais, e um total de 887 trabalhos científicos foram encontrados no banco internacional.

Considerando-se que muitos destes não enfocavam exatamente a temática de interesse, sendo tais descritores somente citados ao longo do texto, um refinamento da busca foi realizado a fim de identificar as publicações que efetivamente relacionavam-se ao tema pesquisado. Dessa maneira, as pesquisadoras leram cada um dos resumos, selecionando aqueles que continham a palavra "sociemotional" no título, resumo e/ou palavraschaves, sendo excluídos todos aqueles que não se relacionavam diretamente à temática pesquisada. Como exemplo pode-se citar trabalhos que foram desconsiderados na base de dados internacional: "Are television and video games really harmful for kids"? "How European, American and Taiwanese mothers talk to their children about learning".
A partir desses critérios, um total de 67 trabalhos foram selecionados e analisados, sendo 17 nacionais e 50 internacionais, classificados em relação às seguintes categorias: ano de publicação, área de conhecimento, tipo de estudo, tema enfocado, instrumento utilizado e população estudada. Os resultados são apresentados a seguir.

\section{Resultados e Discussão}

O construto socioemocional vem sendo investigado desde meados do século XX no cenário internacional (Becker, 1964). Sobretudo no Brasil, esse interesse, apesar de recente, vem aparecendo na literatura nos últimos anos (Abed, 2014; Hutz \& Nunes, 2001; Marturano, Toller \& Elias, 2005; Nunes, Hutz \& Giacomoni, 2009; Santos \& Primi, 2014). Tendo como foco as publicações dos últimos cinco anos, o levantamento dos anos em que os trabalhos foram publicados foi realizado, estando os resultados apresentados no Gráfico 1.

Gráfico 1. Número de publicações nacionais e internacionais a respeito da temática socioemocional, por ano

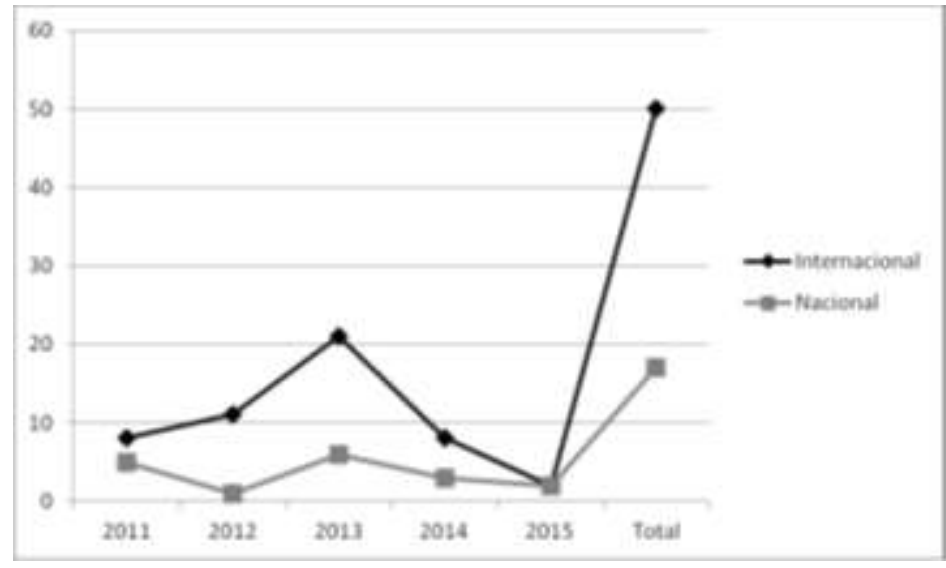

Fonte: Elaborado pelas autoras. 
Pode-se observar no Gráfico 1 que, em relação aos trabalhos nacionais, o número de estudos mostra-se inconstante no período analisado, visto que, de modo geral, um número pequeno de publicações é encontrado em todos os anos analisados. Duas quedas podem ser notadas no período, a primeira acontecendo em 2012, seguida de um pico e, a segunda, em 2014 e 2015. Salienta-se, no entanto, ressalva na interpretação do dado referente à produção de 2015, visto que a busca dessa pesquisa foi realizada em maio do ano citado, de maneira que outros trabalhos podem ter sido publicados posteriormente e não foram analisados.

Tais dados confirmam a constatação de Santos e Primi (2014) ao destacarem que, ainda no Brasil, estudos a respeito desse construto ainda são bastante recentes. Quanto aos trabalhos internacionais, verifica-se um número bem maior de estudos no mesmo período, ainda que importantes oscilações podem ser notadas, com aumento entre os anos de 2011 e 2013, seguido de uma queda nos dois anos seguintes.

Em relação à produção internacional, se considerarmos que foram encontrados 50 trabalhos no período de cinco anos, a média de publicação, no período investigado, é de dez trabalhos por ano, valor que pode ser considerado baixo quando comparado, por exemplo, ao número de pesquisas encontradas sobre os aspectos cognitivos no mesmo período, que apontaram mais de 18 mil artigos abordando esse construto.

Convém, entretanto, salientar que uma das limitações a serem apontadas refere-se a uma dificuldade ressaltada na literatura acerca da utilização de diferentes terminologias para o mesmo construto, fato que pode ter atuado como barreira quando se analisam as terminologias utilizadas, as quais são diversas e podem ser divergentes, conforme destacado por Lipnevich e Roberts (2012). Dadas as palavras-chave utilizadas na busca no banco de dados, considera-se a possibilidade de que alguns estudos não tenham sido localizados devido à seleção destas nos mecanismos de busca.

Posteriormente, os estudos foram analisados considerando-se a área da Psicologia a que pertenciam. A partir dessa análise, foi possível verificar que, a maior parte dos trabalhos internacionais pertenciam à área da Psicologia do Desenvolvimento $(n=31 ; 62 \%)$, confirmando o crescente interesse de pesquisadores em identificar as competências socioemocionais ao longo do processo de desenvolvimento humano, destacando-se sua influência nos aspectos sociais, escolares e laborais (Alzina \& Escoda, 2007; Bzuneck, 2004; Cia, Pamplin, \& Williams, 2008; Osti \& Brenelli, 2013; Paiva \& Boruchovitch, 2010). Tal área englobou quatro trabalhos nacionais (24\% do total analisado).

Uma segunda área bastante enfocada foi a de Educação ( $n=14 ; 28 \%$ nos estudos internacionais e $\mathrm{n}=13,76 \%$ nos estudos nacionais), baseada na hipótese de que os fatores socioemocionais podem influenciar, de maneira considerável, o desempenho acadêmico dos estudantes (Alzina \& Escoda, 2007; Bzuneck, 2004; Cia, Pamplin, \& Williams, 2008). Parte desse interesse pode ser justificado perante a constatação de que, mais recentemente, na busca de como trazer essas competências para a escola, organizações como a Organização para a Cooperação e o Desenvolvimento Econômico (OCDE) passaram a produzir material de apoio para governos e instituições, incentivando-as a criarem políticas e práticas voltadas para a promoção dessas competências.

Consequentemente, uma série de pesquisas nesse contexto têm sido conduzidas, com resultados positivos. Nesse sentido, a literatura tem demonstrado que as competências socioemocionais podem contribuir para o aprimoramento do 
processo de ensino-aprendizagem, promoção do sucesso escolar, prevenção de problemas de aprendizagem, insucesso acadêmico (Abed, 2014), frequência e horas adicionais de estudo (Delaney, Harmon \& Ryan, 2013), menores taxas de abandono escolar, desemprego, envolvimento em crimes e gravidez na adolescência (Santos \& Primi, 2014). Tais constatações justificam o interesse de pesquisadores na influência desse construto na educação (Sabol \& Robert, 2012), assim como a presença de fatores relacionados às competências socioemocionais passou a estar presente no currículo padrão da maioria das escolas dos Estados Unidos, Japão, Áustria, Reino Unido, Finlândia, Coreia, Israel e Singapura, países que dispararam no estudo das competências socioemocionais, ocupando o papel central em seus currículos nacionais (Lipnevich \& Roberts, 2012; Partnership for 21 st Century Skills, 2007, 2008).

Por último, e menos representados, foram encontrados, internacionalmente, trabalhos na área Organizacional $(n=4 ; 8 \%)$ e da Saúde ( $n=1,2 \%)$, os quais, ainda que em menor número, mostram-se importantes dado o reconhecimento de que as competências socioemocionais exercem influência importante em diferentes aspectos relacionados à saúde do indivíduo, bem-estar e funcionamento da vida em geral (Eisenberg, 2006; Goldenberg, Matheson \& Mantler, 2006; Guerra \& Bradshaw, 2008; Lipnevich \& Roberts, 2012; Lopes, Salovey, Coté, Beers \& Petty, 2005).

Especificamente no contexto organizacional, o interesse no construto vem sendo destacado a partir do reconhecimento das vantagens que ele pode exercer nesse ambiente, tais como estabilidade no emprego, confiança, perseverança, melhor adaptação comportamental (Bowles \& Gintis, 1976; Heckman \& Rubinstein, 2001; Heckman, Stixrud \& Urzua, 2006; Klein, Spady \& Weiss, 1991; Lindqvist \&Vestman, 2011; MacCann, Duckworth \& Roberts, 2009; Thiel \& Thomsen, 2013), ainda que, no contexto laboral nacional, a temática ainda esteja sendo pouco explorada.

Em seguida, com a finalidade de compreender os temas que estavam sendo avaliados nesses trabalhos, a tabulação dos dados referentes a esse aspecto foi realizada e indicou a existência de 27 diferentes focos de estudo. Tais temas podem ser agrupados em cinco categorias: 1. Aspectos sociais: interação entre cuidadores e crianças, relações interpessoais, interação entre pares. 2. Características de personalidade: autorregulação, apego, motivação, timidez, afeto, ajustamento socioemocional, autoestima, competência emocional, empatia, esperança, liderança, temperamento. 3. Fatores de risco/proteção desenvolvimental: vulnerabilidade, maus tratos, nível socioeconômico, saúde mental, transtornos emocionais. 4. Indicadores de sucesso/fracasso escolar e profissional: estrutura educacional, identificação de características socioemocionais, institucionalização, sucesso profissional.E 5. Aspectos cognitivos: competências cognitivas, resolução de problemas, superdotação. Os resultados podem ser visualizados na Tabela 1, separados por origem (nacional ou internacional). 
Tabela 1. Temas pesquisados nos estudos nacionais e internacionais sobre competências socioemocionais na Psicologia

\begin{tabular}{|c|c|c|c|c|}
\hline \multirow{2}{*}{ Tema } & \multicolumn{2}{|c|}{ Nacional } & \multicolumn{2}{|c|}{ Internacional } \\
\hline & Frequência & $\%$ & Frequência & $\%$ \\
\hline Interação entre cuidadores e crianças & - & - & 10 & 20,0 \\
\hline Autorregulação & 2 & 11,0 & 9 & 18,0 \\
\hline Relações interpessoais & - & - & 3 & 6,0 \\
\hline Apego & - & - & 2 & 4,0 \\
\hline Motivação & - & - & 2 & 4,0 \\
\hline Timidez & - & - & 2 & 4,0 \\
\hline Vulnerabilidade & - & - & 2 & 4,0 \\
\hline Afeto & - & - & 1 & 2,0 \\
\hline Ajustamento socioemocional & - & - & 1 & 2,0 \\
\hline Autoestima & 3 & 17,0 & 1 & 2,0 \\
\hline Competências cognitivas & - & - & 1 & 2,0 \\
\hline Competência emocional & - & - & 1 & 2,0 \\
\hline Empatia & - & - & 1 & 2,0 \\
\hline Esperança & - & - & 1 & 2,0 \\
\hline Estrutura educacional & - & - & 1 & 2,0 \\
\hline Identificação de características socioemocionais & - & - & 1 & 2,0 \\
\hline Institucionalização & - & - & 1 & 2,0 \\
\hline Interação entre pares & - & - & 1 & 2,0 \\
\hline Liderança & - & - & 1 & 2,0 \\
\hline Maus tratos & - & - & 1 & 2,0 \\
\hline Nível socioeconômico & - & - & 1 & 2,0 \\
\hline Resolução de problemas & - & - & 1 & 2,0 \\
\hline Saúde mental & - & - & 1 & 2,0 \\
\hline Sucesso profissional & - & - & 1 & 2,0 \\
\hline Superdotação & - & - & 1 & 2,0 \\
\hline Temperamento & - & - & 1 & 2,0 \\
\hline Transtornos emocionais & - & - & 1 & 2,0 \\
\hline Desempenho acadêmico & 7 & 42,0 & - & - \\
\hline Emoção & 1 & 6,0 & - & - \\
\hline Estrutura educacional & 1 & 6,0 & - & - \\
\hline Personalidade & 1 & 6,0 & - & - \\
\hline Pensamento crítico & 1 & 6,0 & - & - \\
\hline Ausência de tema explicitado no resumo & 1 & 6,0 & - & - \\
\hline Total & 17 & 100,0 & 50 & 100,0 \\
\hline
\end{tabular}

Fonte: Elaborada pelas autoras.

Ao se comparar os contextos nacional e internacional, importantes diferenças são percebidas. Em relação ao primeiro citado, verifica-se que os estudos se voltam, prioritariamente, à investigação de sete diferentes aspectos, com predomínio daqueles relacionados ao desempenho acadêmico, fazendo-se 
notar, ainda, a dificuldade de acesso ao tema de um dos estudos, cuja informação não se encontrava disponibilizada no resumo. Por outro lado, no contexto internacional, 27 diferentes temas foram foco de estudo dos pesquisadores, havendo predominância na investigação da interação entre cuidadores e crianças e autorregulação. Comparando-se os dois contextos, pode ser verificada a diferença em relação ao número de temas investigados (muito maior internacionalmente), assim como no interesse dos pesquisadores, considerando-se que os temas mais investigados em cada cenário são diferentes, havendo, na maior parte dos casos, temas investigados internacionalmente que não foram enfocados nos estudos nacionais e vice-versa.

Posteriormente, a categoria tipo de estudo foi analisada. Os resultados mostraram que a maior parte dos trabalhos nacionais era de natureza empírica ( $\mathrm{n}=13 ; 76 \%$ ), havendo também importante número de trabalhos teóricos $(n=4 ; 24 \%)$. O mesmo quadro foi encontrado nos trabalhos internacionais $(n=39 ; 78 \%$ de trabalhos empíricos e $n=11 ; 22 \%$ teóricos). Tal constatação confirma os dados da literatura acerca do tipo de estudo que tem sido priorizado na investigação das competências socioemocionais (Barbarin, 2013; Lecannelier, Silva, Melo \& Morales, 2014; McCoy \& Raver, 2011).

Ao verificar que a maior parte dos estudos possuía natureza empírica, analisou-se, dentre estes, os instrumentos que foram utilizados pelos pesquisadores. Destaca-se o fato de que, para esse levantamento, caso a informação acerca do instrumento não se encontrasse disponibilizada no resumo, sempre que possível, o acesso ao trabalho completo foi realizado. Tal procedimento se mostrou necessário a fim de que se pudesse identificar o nome do instrumento utilizado, visto que, algumas vezes, tal dado era citado de maneira geral no resumo (por exemplo: utilizou-se um questionário para avaliar as competências socioemocionais). Os resultados encontram-se disponibilizados na Tabela 2.

Tabela 2. Instrumentos utilizados nos estudos empíricos na investigação da temática competência socioemocional

\begin{tabular}{lcc}
\hline Instrumentos encontrados nos estudos internacionais & $\mathbf{N}$ & $\mathbf{\%}$ \\
\hline Infant-Toddler Social Emotional Assessment & 2 & 12,0 \\
Questionnaire & 2 & 12,0 \\
Attachment and Biobehavioral Catch-up Intervention & 1 & 6,0 \\
Child Behavior Checklist & 1 & 6,0 \\
Child Social Surrogate Questionnaire (CSSQ) & 1 & 6,0 \\
Early Childhood Environment Rating Revisado-Scale & 1 \\
Early Childhood Longitudinal Study-Kindergarten Cohort (ECLS-K) & 1 \\
NICHD Study of Early Child Care and Youth Development & 1 \\
Parent Demographic Questionnaire & 1 \\
Parenting Interactions with Children - Checklist of Observations Linked to Outcomes (PICCOLO) & 1 \\
PCL-R & 1 \\
Social-Emotional Scale for Adaptive Behavior Assessment System (ABAS-II) & 6,0 \\
TULSA & 6,0 \\
Wmatrix & 6,0 \\
Dictionary of Affect and Language (DAL) & 1
\end{tabular}


Adaptación Socioemocional en escolares

Cuestionario de Adaptación Socioemocional

Cuestionario de Motivaciones e Intereses

Cuestionario de Comportamientos Socialmente Responsables

Teenage Inventory of Social Skills

Texas Social Behavior Inventory
$1 \quad 16,6$

16,6

$1 \quad 16,6$

$1 \quad 16,6$

$1 \quad 16,6$

$1 \quad 16,6$

Fonte: Elaborada pelas autoras.

$\mathrm{Na}$ Tabela 2, verifica-se que, nos artigos empíricos selecionados para estudo, foram encontrados 23 diferentes instrumentos, sendo 17 nos trabalhos provenientes da base de dados internacional e seis das bases de dados nacionais. Importante destacar que enquanto alguns estudos fizeram uso de mais de um instrumento, outros citaram, apenas de forma geral, o uso de algum recurso (por exemplo: questionário).

Faz-se notar maior amplitude de instrumentos utilizados nas pesquisas encontradas nas bases internacionais, ainda que somente dois apresentem, diretamente, em seu título o construto foco: Infant-Toddller Social Emotional Assessment e Social-Emotional Scale for Adaptive Behavior Assessment System. Os demais avaliam construtos que podem estar relacionados à temática.

Em relação aos instrumentos utilizados nas pesquisas provenientes das bases nacionais, convém salientar que somente duas pesquisas fizeram uso de instrumento, sendo de autores internacionais e conduzidas no Chile. Uma delas fez uso de um instrumento específico (Adaptación Socioemocional en escolares) e, a outra, de cinco diferentes instrumentos, sendo que somente um relacionava-se diretamente à investigação das competências socioemocionais (Cuestionario de Adaptación Socioemocional). Nenhuma pesquisa brasileira, empírica, fazendo uso de instrumento foi encontrada nas bases consultadas.
A verificação da utilização de 23 diferentes instrumentos, ainda que em um primeiro momento tal dado pareça indicar um número razoável, deve ser interpretada com cautela. Um levantamento internacional realizado por Gokiert, Georgis, Tremblay, Krishnan, Vandenberghe e Lee (2014) de medidas de avaliação socioemocional apontou a existência de 78 instrumentos voltados à infância, tendo como critério a disponibilidade na língua inglesa, dentro de um processo de busca que envolveu bases de dados científicas, sites das editoras e compêndios de medidas socioemocionais. Do mesmo modo, Santos e Primi (2014) identificaram 113 instrumentos que se apresentavam associados a alguma medida de resultado relacionada ao bem-estar, classificando-os em medidas de personalidade, habilidades sociais, temperamento, motivação e interesses profissionais.

Diante desses dados, o número de instrumentos disponíveis aponta para a importância reconhecida desse tipo de competência, cujas formas de avaliação disponíveis se mostraram adequadas e com comprovadas evidências psicométricas. Entretanto, a situação no Brasil se mostra bem diferente. O país não possui instrumento específico para uso profissional certificado pelo Conselho Federal de Psicologia. Até o momento, o único instrumento encontrado na literatura é o Senna, questionário socioemocional aplicável a alunos do $5^{\circ}$ ano do Ensino Fundamental ao $3^{\circ}$ ano do Ensino Médio, tendo como indicadores as atitudes 
observáveis em sala de aula (Santos \& Primi, 2014). Diferentes pesquisas foram conduzidas com o instrumental e encontram-se disponibilizadas sob a forma de um relatório on-line (http://educacaosec21.org.br/wpcontent/uploads/2013/07/desenvolvimentosocioemocional-e-aprendizado-escolar.pdf), cujo desenvolvimento tem, como objetivo principal, o subsídio tanto de políticas públicas quanto de práticas pedagógicas.

Como consequência dessa lacuna, importantes prejuízos podem estar sendo gerados, notadamente se considerarmos que a detecção precoce, por meio de avaliação ou sondagem, dos estudantes em risco de desenvolvimento de dificuldades socioemocionais pode guiar os programas de intervenção, com foco no desenvolvimento saudável. $\mathrm{Na}$ área acadêmica, especificamente na área de avaliação psicológica, o número ainda muito reduzido de pesquisas realizadas, assim como a ênfase que se tem feito notar, na investigação dos aspectos cognitivos apontam para uma lacuna que precisa ser sanada pelos pesquisadores, dada a ênfase que as competências socioemocionais vem alcançando, notadamente diante dos resultados educacionais e laborais provenientes da sua promoção. Mais especificamente, o desenvolvimento de instrumentos de medida desse construto, adaptados ou construídos com base nas características dos brasileiros, se faz essencial para a avaliação das iniciativas que vêm sendo, progressivamente, adotadas por diferentes instituições e governos estaduais (Nakano \& Santos, no prelo).

Sendo assim, o franco desenvolvimento nessa área aponta a necessidade de olharmos para esse campo também no Brasil. Isso porque, apesar do reconhecimento crescente da importância de uma educação mais plena e mais abrangente que envolva o ser humano em sua integralidade, nota-se que a maior parte dos instrumentos utilizados na avaliação escolar e dos sistemas educacionais ainda é voltada basicamente à investigação do aspecto cognitivo, medido por meio de testes de desempenho (Abed, 2014, Major \& Seabra-Santos, 2013, Santos \& Primi, 2014).

Por último, a amostra envolvida nos estudos foi categorizada, considerando-se a fase de desenvolvimento dos participantes. Os resultados mostraram que nos estudos nacionais a população infantil é mais estudada $(n=8 ; 42,1 \%)$, seguida de adolescentes $(n=7 ; 36,8 \%)$ e adultos $(n=3 ; 15,8 \%)$. Um estudo não informou de modo claro a amostra utilizada. Internacionalmente, a população infantil também é a mais investigada $(n=32 ; 60 \%)$, seguida dos adultos $(n=16 ; 30 \%)$, recém-nascidos $(n=3 ; 6 \%)$ e adolescentes $(n=2 ; 4 \%)$. Os dados podem ser visualizados na Tabela 3 .

Tabela 3. População estudada nos trabalhos nacionais e internacionais na temática socioemocional

\begin{tabular}{llc}
\hline População & Nacional & Internacional
\end{tabular}




\begin{tabular}{lcccc}
\hline & $\mathbf{N}$ & $\mathbf{0}$ & $\mathbf{N}$ & $\mathbf{\%}$ \\
\cline { 2 - 5 } Recém-nascido & - & - & 3 & 6,0 \\
Crianças & 8 & 42,1 & 32 & 60,0 \\
Adolescentes & 7 & 36,8 & 2 & 4,0 \\
Adultos & 3 & 15,8 & 16 & 30,0 \\
Não informada & 1 & 5,3 & - & - \\
\hline Total & $\mathbf{1 9}$ & $\mathbf{1 0 0 , 0}$ & $\mathbf{5 3}$ & $\mathbf{1 0 0 , 0}$ \\
\hline
\end{tabular}

Fonte: Elaborada pelas autoras.

Os dados apontam para o interesse predominante dos pesquisadores, tanto nacionais quanto internacionais, na investigação das competências socioemocionais na infância. De acordo com MacCann, Duckworth e Roberts (2009), essa preferência se justifica diante das evidências de que se essas competências cognitivas forem avaliadas durante a infância e adolescência desempenharão um papel crucial na prevenção do absenteísmo escolar, bem como infrações disciplinares. Assim, tem-se visto maior interesse de investigação na população de crianças e adolescentes, notadamente no contexto escolar, com objetivo de analisar o desenvolvimento e identificar quais estratégias podem ser utilizadas visando ao sucesso na vida pessoal e profissional (Osti \& Brenelli, 2013).

De acordo com Abed (2014), estudos mostraram que, na pré-escola, variáveis relacionadas à personalidade predizem conquistas individuais na formação acadêmica futura. Já para crianças e adolescentes, fatores psicossociais, tais como autoeficiência, autoconceito e confiança, mostraramse bons preditores do desenvolvimento em leitura, ciências e matemática em várias avaliações nacionais e internacionais de larga escala. Também Sabol e Robert (2012) verificaram que crianças com impedimentos na aquisição de tais competências apresentam um risco aumentado de dificuldades comportamentais quando iniciam a primeira série, de maneira que, segundo os autores, a detecção precoce de pontos fortes e fracos podem garantir a melhor via de desenvolvimento da criança, prevenindo o surgimento de dificuldades emocionais sérias e comportamentos antissociais durante a adolescência. Tomando-se essa postura preventiva da avaliação e estímulo às competências socioemocionais, o interesse precoce se justifica.

\section{Considerações Finais}

De modo geral, o objetivo geral desta pesquisa, de efetuar a análise da produção científica nacional e internacional a respeito do construto competência socioemocional, foi atingido. A identificação de suas tendências e lacunas possibilitou, ao longo do texto, debater e mapear a produção acadêmica do presente construto, notadamente por meio da classificação dos estudos em relação a aspectos específicos, tais como ano de publicação, área de conhecimento, tipo de estudo, tema enfocado, instrumentos utilizados e população estudada.

Os resultados mostraram que as competências socioemocionais são bastante valorizadas e investigadas no contexto internacional, o qual conta com importante número de pesquisas, de instrumentos de avaliação e de políticas públicas voltadas a esses aspectos. De modo oposto, nacionalmente, o construto ainda tem se mostrado muito recente e pouco explorado nas pesquisas brasileiras, seja na prática escolar, nas pesquisas acadêmicas, políticas públicas e intervenções com objetivo de promovê-las (Santos \& Primi, 2014). 
Entretanto, a revisão também mostrou que, ainda de maneira tímida, vem ganhando destaque e motivando o interesse de pesquisadores.

Cautela é recomendada na interpretação dos resultados aqui apresentados, visto que somente cinco bases de dados eletrônicas foram consultadas, limitando-se o período da consulta aos últimos cinco anos. Do mesmo modo, a seleção dos descritores utilizados nas buscas pode ter exercido influência importante nos resultados, visto que, conforme já citada, a ausência de consenso em relação à terminologia ainda se faz notar entre os pesquisadores.

Por fim, os achados da presente pesquisa revelam que essa área se apresenta promissora ao crescimento, tanto internacionalmente quanto nacionalmente. Nesse sentido, a análise da produção científica, como foi realizada neste estudo, mostra-se como uma necessidade para a tomada de decisão quanto aos rumos da própria ciência e das políticas científicas e tecnológicas, áreas que merecem maior atenção e generalização dos resultados produzidos Joly, Martins, Abreu, Souza \& Cozza, 2004; Witter, 1999), justificando a condução de estudos dessa natureza, dado o recente e intenso interesse pelo tema e a ausência de estudos do tipo estado da arte no Brasil.

Fez-se notar, diante do reconhecimento das competências socioemocionais como elementos necessários ao sucesso acadêmico, profissional e pessoal e sua importância nas mais diferentes áreas do conhecimento, que esforços na Psicologia se fazem necessários. Tal situação se fortalece diante da constatação de que um de seus movimentos mais atuais, o da Psicologia Positiva, tem se pautado na busca pela valorização de características relacionadas a aspectos positivos do indivíduo. Tais discussões se mostram passíveis de serem ampliadas, por exemplo, por meio do desenvolvimento e incentivo às competências socioemocionais.

\section{Referências}

Abed, A. L. Z. (2014, maio 19). O desenvolvimento das habilidades socioemocionais como caminho para a aprendizagem e o sucesso escolar de alunos da educação básica. Recuperado em 15 fevereiro, 2015, de http://educacaosec21.org.br/wpcontent/uploads/2013/08/habilidadessocioemocionais_CNE.pdf

Alzina, R. B., \& Escoda N. P. (2007). Las competências emocionales. Educación XXI, 10, 61-82.

Barbarin, O. (2013). A Longitudinal Examination of Socioemotional Learning in African American and Latino Boys Across the Transition From Pre-K to Kindergarten. American Journal of Orthopsychiatry, 83(2,3), 156-164.

Becker, G. (1964). Human Capital: a Theoretical and Empirical Analysis, with Special Reference to Education. New York: Columbia University Press.

Berger, C., Milicic, N., Alcalay, L., \& Torretti, A. (2014). Programa para el Bienestar y Aprendizaje Socioemocional en estudiantes de tercero y cuarto grado: descripción y evaluación de impacto. Revista Latinoamericana de Psicologia,46(3), 169-177.

Bowles, S., \& Gintis, H. (1976). Schooling in Capitalist America. New York: Basic Books.

Bzuneck, J. A. (2004). A motivação do aluno, Aspectos introdutórios. In E. Boruchovitch \& J. A. Bzuneck (Orgs.). Motivação do Aluno: contribuições da psicologia contemporânea (pp. 0926). Petrópolis: Vozes. 
Cia, F., Pamplin, R. C. O., \& Williams, L. C. A. (2008). O impacto do envolvimento parental no desempenho acadêmico de crianças escolares. Psicologia em Estudo, 13(2), 351-360.

Delaney, L., Harmon, C., \& Ryan, M. (2013). The Role of Noncognitive Traits in Undergraduate Students Behaviours. Economics of Education Review, 32, 181-195.

Durlak, J. A., Weissberg, R. P., Dymnicki, A. B., Taylor, R. D., \&Schellinger, K. B. (2011). The impact of Enhancing Student's Social and Emotional Lerarning: a Meta-Analysis of School-Based Universal Interventions. Child Development, 82(1), 405-432.

Gokiert, R. J., Georgis, R., Tremblay, M., Krishnan, V., Vandenberghe, C., \& Lee, C. (2014). Evaluating the Adequacy of Social-Emotional Measures in Early Childhood. Journal of Psychoeducational Assessment, 9, 1-14.

Heckman, J. J., \& Rubinstein, Y. (2001). The Importance of Noncognitive skills: Lessons from the GED Testing Program. American Economic Review, 91, 145-149.

Heckaman, J. J., Stixrud, J., \& Urzua, S. (2006). The Effect of Cognitive and Noncognitive Abilities on Labor Market Outcomes and Social Behavior. Journal of Labor Economics, 24(3), 411-482.

Hutz, C. S., \& Nunes, C. H. S. S. (2001). Escala Fatorial de Neuroticismo. São Paulo: Casa do Psicólogo.

Joly, M. C. R., Martins, R. X., Abreu, M. C., Souza, P. R. R., \& Cozza, H. F. P. Análise da produção científica em avaliação psicológica informatizada. Avaliação Psicológica, 3(2), 121 129, 2004.

Nunes, C. H. S. S., Hutz, C. S., \& Giacomoni, C. H. (2009). Associação entre bem-estar subjetivo e personalidade no modelo dos cinco grandes fatores. Avaliação Psicológica, 8(1), 99-108.
Lee, J., \& Shute, V. J. (2009). The Influence of Noncognitive Domains on Academic Achievement in K-12. Princeton: Educational Testing Service. Lecannelier F., Silva, J. R., Melo, M. H. R., \& Morales R. (2014). Effects of an Intervention to Promote Socioemotional Development in Terms of Attachment Security: A Study in Early Institutionalization in Chile. Infant Mental Health Journal, 35(2), 151-159.

Lindqvist, E., \&Vestman, R. (2011). The Labor Market Returns to Cognitive and Noncognitive Ability: Evidence from the Swedish Enlistment. American Economic Journal Applied Economics, 3(1), 101-128.

Lipnevich, A. A., \& Roberts, R. D. (2012). Noncognitive Skills in Education: Emerging Research and Applications in a Variety of International Contexts. Journal of Psychology and Education, 2(2), 173-177.

Major, S., \& Seabra-Santos M. J. (2013). Uso de inventários comportamentais para avaliação socioemocional em idade pré-escolar. Avaliação Psicológica, 12(1), 101-107.

Marturano, E. M., Toller, G. P., \& Elias, L. C. S. (2005). Gênero, adversidade e problemas socioemocionais associados à queixa escolar. Estudos de Psicologia Campinas, 22(4), 371-380.

Maccann, C., Duckworth, A., \& Roberts, R. D. (2009). Identifying the Major Facets of Conscientiousness in High School Students and their Relationships with Valued Educational Outcomes. Learning and Individual Differences, 19, 451-458.

Mccoy, D. C., \& Raver, C. C. (2011). Emotional Expressiveness, Child Emotion Regulation, and Child Behavior Problems among Head Start Families. Social Development, 20(4), 741761. 
Mervielde, I., Deary, J., DeFruyt F., \& Ostendorf F. (1999). Construction of the Hierarchical Personality Inventory for Children (HiPIC). Trabalho apresentado no Proceedings of the Eight European Conference on Personality Psychology. Tilburg, Tilburg University Press. Messick, S. (1979). Potential Uses of Noncognitive Measurement in Education. Journal of Educational Psychology, 71, 281-292.

Nunes, C. H. S. S., Hutz, C. S., \& Giacomoni, C. H. (2009). Associação entre bem-estar subjetivo e personalidade no modelo dos cinco grandes fatores. Avaliação Psicológica, 8(1), 99-108.

Paiva, M. L. M. F., \& Boruchovitch, E. (2010). Orientações motivacionais, crenças educacionais e desempenho escolar de estudantes do ensino fundamental. Psicologia em Estudo Maringá, 15(2), 381-389.

Osti, A., \& Brenelli, R. P. (2013). Sentimentos de quem fracassa na escola: análise das representações de alunos com dificuldades de aprendizagem. Psico-USF, 18(3), 417-426.

Sabol, T. J., \& Robert C. P. (2012). Patterns of School Readiness Forecast Achievement and Socioemotional Development at the End of Elementary School. Child Development, 83(1), 282-299.

Santos, D., \& Primi R. (2014, maio 19). Desenvolvimento socioemocional e aprendizado escolar: Uma proposta de mensuração para apoiar políticas públicas. Educação para o Século XXI. 2014. Recuperado de http://educacaosec21.org.br/wpcontent/upl oads/2013/07/RioReportAv13.pdf.

Stankov, L. (2013). Non Cognitive Predictors of Intelligence and Academic Achievement: an
Important Role of Confidence. Personality and Individual Differences, 55, 727-732.

Simões, F., \& Alarcão, M. (2011). A eficácia da mentoria escolar na promoção do desenvolvimento socioemocional e instrumental de jovens. Educação e Pesquisa, 37(2), 339-354.

Thiel, H., \& Thomsen, S. L. (2013). Non Cognitive Skills in Economics: Models, Measurement and Empirical Evidence. Research in Economics, 67, 189-214.

Witter, G. P.. Metaciência e leitura. In G. P. Witter (Org.). Leitura: textos e pesquisas (pp. 13-22) Campinas: Alínea, 1999.

Recebido em 14/07/2016

Aprovado em 20/07/2017 